\section{Gonorrhö bald unheilbar?}

\section{Tripper auf} Resistenztrip

- Ein japanisch-schwedisches Forscherteam hat einen neuen multiresistenten Stamm von Neisseria gonorrhoeae entdeckt. Der Stamm mit dem Namen H041 wurde aus dem Pharynx einer Prostituierten im japanischen Kyoto isoliert und ist unempfindlich gegen so gut wie alle verfügbaren Antibiotika, also auch gegen Cephalosporine wie Ceftriaxon, die bisher als letzter Ausweg gegolten haben. Ursache der Ceftriaxon-Resistenz ist ein neues Allel des penA-Gens.

Um die Ausbreitung der Cefixim- und Ceftriaxon-Resistenz wenigstens zu verlangsamen, sind laut Onishi die Bemühungen um Prävention und Kontrolle der Gonorrhö weltweit zu verstärken. Und schließlich müssten auch effektive neue Medikamente, ob einzeln oder in Kombination verabreicht, gegen die Erkrankung gefunden werden.

Dr. Robert Bublak

\section{Online-Foren zu Prostatakrebs} Nützlich oder gefährlich?

\begin{abstract}
— Die Bedeutung des Internets als Informations- und Beratungsquelle nimmt stetig zu. Da Patienten mit einem lokal begrenzten Prostatakrebs mehrere Therapieoptionen offen stehen, haben Heidelberger Urologen untersucht, welchen Stellenwert der Austausch unter Patienten in Online-Foren hat [Huber J. et al. BJU Int 2011, 107: 1570-5].

Über 32 Monate verfolgten sie insgesamt 500 Threads im Forum der größten OnlineSelbsthilfegruppe für Prostatakrebs in Deutschland (www.forum-prostatakrebs-bps.de). Ausgewertet wurden 83 Threads mit insgesamt 1.630 Einzelbeiträgen.

Die Patienten bekamen in den Online-Foren Infos, Ratschläge und vor allem emotionale Unterstützung, so das Ergebnis. Der Austausch in den Foren führe dazu, dass sich die Patienten aktiv mit ihrer Krankheit auseinandersetzen, sie würden beruhigt und dazu motiviert, weitere Infos einzuholen, so die Autoren.

Die Nutzer rieten häufiger zu einer Strahlentherapie als zu chirurgischen Maßnahmen. In mehr als der Hälfte der Beiträge wurden bestimmte Ärzte, in 36\% der Fälle bestimmte Universitätskliniken empfohlen. Insgesamt trage diese Unterstützung zur Krankheitsbewältigung bei, so die Wissenschaftler.

In etwa der Hälfte der Fälle empfahlen Betroffene, eine Zweitmeinung zum Biopsieresultat einzuholen oder zusätzliche bildgebende Verfahren. Dies sei aus medizinischer Sicht aber nicht immer sinnvoll gewesen, bemängeln die Autoren. Einen möglichen negativen Effekt sieht der Urologe auch darin, dass die Beiträge in den Foren häufig von nur einigen wenigen Betroffenen verfasst werden. Deren Meinung beeinflusse somit sehr viele Infosuchende. Derartige Negativeffekte könnten aber durch das Arzt-Patienten-Gespräch ausgeräumt werden. Hier fungiere der Arzt als Korrektiv.
\end{abstract}

\title{
Radikale Prostatektomie
}

\section{Wie lang soll der PSA-Wert postoperativ kontrolliert werden?}

— Die Ergebnisse einer US-amerikanischen Studie legen nahe, nach radikaler Prostatektomie mit einem Gleason-Score $\leq 6$ auf die jährlichen PSA-Kontrollen zu verzichten, wenn der PSA-Wert nach über zehn Jahren noch immer unter $2 \mathrm{ng} / \mathrm{ml}$ liegt [Loeb et al. J Urol 2011; 186; 500-5]. Gleichzeitig kann man den Patienten beruhigen, denn dies gilt als Hinweis darauf, dass vom operierten Karzinom keine Metastasen ausgegangen sind.

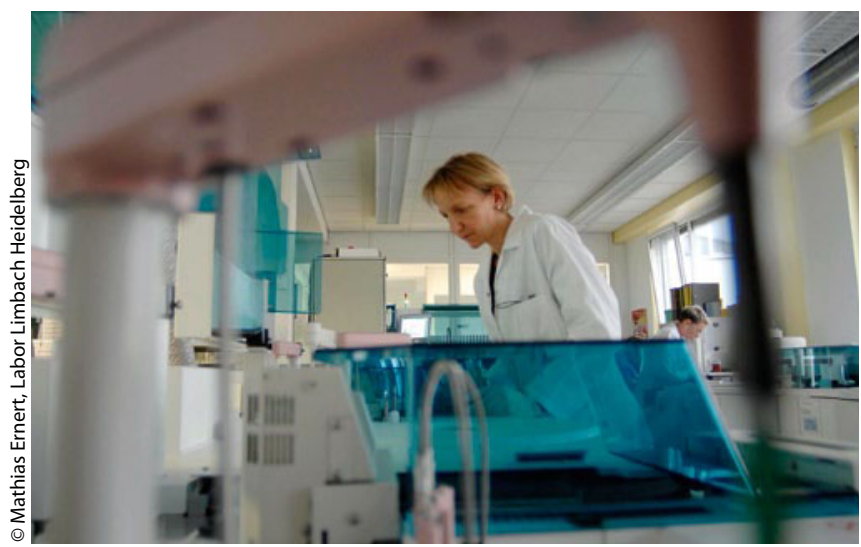

Dle PSA-Bestimmung ist nach mehr als zehn Jahren postoperativ nicht immer erforderlich.
In der Studie wurden 10.609 Prostatakarzinom-Patienten postoperativ mindestens zehn Jahre lang beobachtet. Die Häufigkeit einer Metastasierung sowie die Überlebenszeit korrelierten dabei klar mit dem Zeitpunkt des biochemischen Rezidivs. War der PSA-Wert nach zehn Jahren noch unauffällig, hing der weitere Verlauf allerdings auch vom Stadium der Krebserkrankung zum Zeitpunkt der Operation und der Klassifizierung des Tumors ab. Eine Subgruppe mit 1.583 Männern, die mehr als zehn Jahre ohne Hinweis auf ein PSA-Rezidiv waren, wurde zum Teil bis zu 20 Jahre postoperativ beobachtet. Bei 6,3\% der Operierten erhöhte sich nach den üblichen zehn Beobachtungsjahren der PSA-Wert. Lag der Gleason-Score zum Zeitpunkt der Operation unter 6, waren die Patienten auch 20 Jahre postoperativ noch metastasenfrei.

Die Gefahr für ein PSA-Rezidiv ist der Studie zufolge in den ersten Jahren nach einer radikalen Prostatektomie am höchsten und nimmt dann immer mehr ab. Je früher die Marker-Konzentration wieder steigt, desto kürzer ist die metastasenfreie Zeit und die verbleibende Lebenserwartung des Patienten. Da ein PSA-Wert, der sich die ersten zehn Jahre unterhalb der Nachweisgrenze bewegt hat, nur selten danach noch einmal ansteigt, könnte die Frequenz der PSA-Tests in dieser Patientengruppe verringert werden. Mehr noch: Bei Männern, deren Gleason-Score bei der Prostatektomie $\leq 6$ war und/oder die nur noch eine geringe Lebenserwartung haben, halten die Autoren weitere Kontrollen für unnötig. Diese Patienten gelten als geheilt.

Dr. Christine Starostzik 